\title{
Analysis of MIMO-OFDM: Effect of Mutual Coupling, Frequency Response, SNR and Channel Capacity
}

\author{
Karthik Kumar Vaigandla ${ }^{1^{*}}$, RadhaKrishna Karne ${ }^{2}$, Allanki Sanyasi Rao ${ }^{3}$ \\ ${ }^{1}$ Assistant Professor, ${ }^{2}$ Assistant Professor, ${ }^{3}$ Associate Professor \\ ${ }^{1,2,3}$ Balaji Institute of Technology and Science, Dept. of Electronics \& Communication \\ Engineering, Warangal, Telangana, India \\ ${ }^{1}$ vkvaigandla@bitswgl.ac.in, ${ }^{2} k r k . w g l @ g m a i l . c o m,{ }^{3}$ asr.balajigroup@gmail.com
}

\begin{abstract}
Systematic analysis of systems with Multiple Inputs and Multiple Outputs (MIMO) is presented in this paper. MIMO systems and various techniques for decoupling are discussed, including their effect on performance. As wireless communications systems progress, the challenge is to deliver high-data-rate access with good quality of service (QOS). MIMO technology can also offer greater reliability by boosting the link due to increased spectral efficiency; this can be met by incorporating spatial multiplexing gains and antenna diversity gains. OFDM signals can be used with antenna arrays at both transmitter and receiver for maximum efficiency. As a result, we have MIMO-OFDM. A generalized predictive control for MIMO systems is considered in this paper. Stability and performance of the closed-loop system are measured by using frequency response indicators.
\end{abstract}

Keywords: Multiple Inputs and Multiple Output (MIMO), Orthogonal Frequency Division Multiplexing (OFDM), BER, high data rate, Mutual coupling (MC), channel capacity.

\section{INTRODUCTION}

Wireless communication nowadays requires a high level of speed and throughput. Wireless communications have grown in popularity since high-speed wired communications became popular decades ago. Most wireless networks communicate downlink using OFDM. Adaptable channels make them ideal for use with difficult channels. The use of OFDM for $4 \mathrm{G}$ communications is a great choice. However, OFDM's PAPR is its main disadvantage. There are several methods for reducing the PAPR [21]. Wireless communication has been revolutionized by MIMO technology, which increases transmission capacity without requiring significantly higher antenna power [1]. MIMO technology uses multiple antennas to transmit and receive data in a wireless system. In the past few decades, MIMO has gained widespread popularity because of its performance-enhancing capabilities [2]. Using MIMO systems, both the transmitter and the receiver take advantage of space (or antenna) diversity in order to increase the spectral efficiency. Multiple data streams can be transmitted simultaneously by MIMO systems, improving capacity [3].

Massive MIMO is widely used in modern telecommunications, including long-term evolution (LTE) and wireless local area networks (WLAN) [4]. It is considered a key enabler for fifthgeneration (5G) communications [5] - The massive MIMO system is believed to be a key 
enabler for the 5fth-generation (5G) communications [5]-[7]. Neither mobile terminals nor base stations (BS) can utilize large antennas due to space restrictions and aesthetic reasons. Due to their proximity, antenna elements experience (electromagnetic) mutual coupling. MIMO antennas have mutual coupling due to free space radiation, surface currents, and surface waves. Arrays can be classified in the first two ways, whereas micro strip antennas are more commonly categorized in the third way. An adaptive array's signal-to-interferencenoise ratio (SINR) and the convergence of array signal processing algorithms can be seriously affected by mutual coupling [8], [9]. By causing errors in carrier frequency offset [10], channel estimation [11], and angle of arrival [12], one can degrade these estimations.

\section{SYSTEM MODEL}

\subsection{MIMO System}

MIMO models must include antenna system parameters as a component of the model to account for antenna effects. By using impedance matrices, this can be achieved. In addition to the transmitting and receiving antenna impedances, feeding and loading impedances are also effective parameters to assess the antenna systems. In practice, however, the concept of impedance matrix is more difficult to apply to wave propagation problems directly when it was designed originally for lumped circuit elements [13]. In this approach, one is able to gain some insight into MIMO system performance. MIMO was created by Greg Raleigh in 1996. Using space signs that sway off articles and take different approaches, he demonstrated that recognizable data streams could be sent all at once on a similar repeat. Using OFDM change would be the most sensible approach to meeting the planning requirements of MIMO at higher rates, made by Raleigh [18].

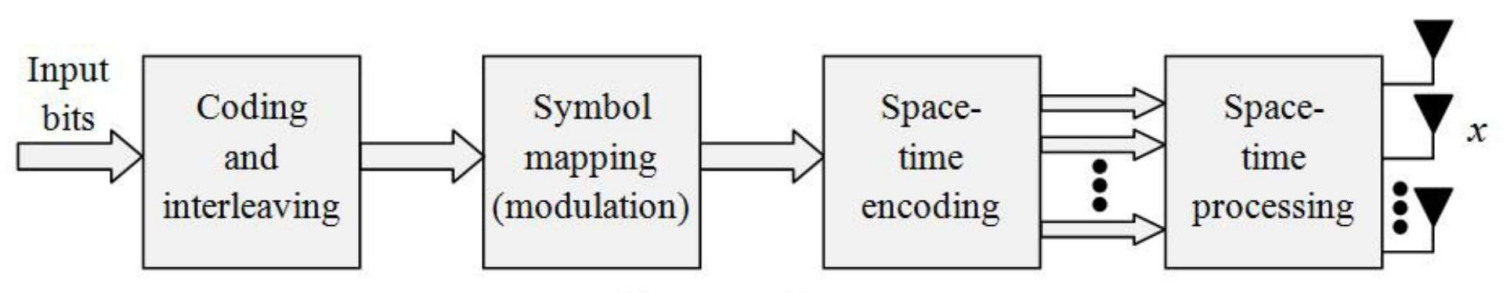

\section{Transmitter}

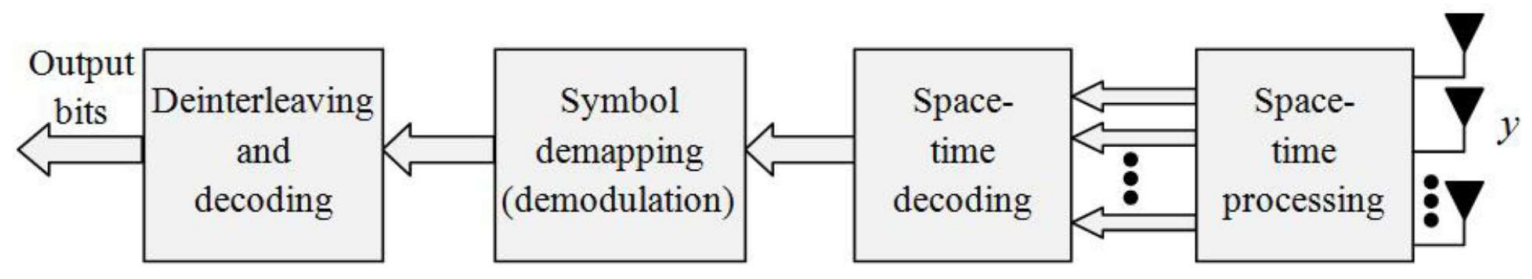

\section{Receiver}

Figure 1. MIMO System

An $\mathrm{N}_{t}$ transmit antenna and a $\mathrm{N}_{\mathrm{r}}$ receive antenna is considered in a narrowband MIMO wireless communication system. Different antenna elements are fed with different streams of data in a system that uses spatial multiplexing signaling. An input-output relation for MIMO wireless systems is based on both transmitting and receiving antenna systems. 
With MIMO you can create a kind of spatial multiplexing by routing many signals between transmitters and receivers. Various channels are measured independently via their transfer parameters, such as channel numbers $\alpha_{11}, \alpha_{12}$, etc. to create a matrix $H$ that describes the transmission function for the various channels. By implementing a transfer matrix, the transfer capacity of the transmission system can be optimized by the system itself, in addition to enhancing diversity. When a MIMO system is designed optimally with a high separation between the signals, it is the diagonal elements of matrix $H$ that are dominant, and in the design of a practical Internet I should strive to achieve this. Matrix $\mathrm{H}$ with more orthogonality results in more decoupling between MIMO channels, and hence, a higher system transfer rate. Receiving antennas respond slightly differently to the signals sent from transmitters. The signal received by a receiver antenna $R_{x 1}$ from a transmitter antenna $T_{x 1}$ is described in $\alpha_{11}$; similarly $\alpha_{21}$ describes the signal from a transmitter antenna $T_{x 1}$ to a receiver antenna $R_{x 2}$, and so on, compiling the matrices $\mathrm{H}$ for transmission and reception respectively [14].

\subsection{MIMO - OFDM System}

The MIMO-OFDM technology has currently been identified as one of the most competitive technologies for the development of future mobile wireless networks. A combination of MIMO and OFDM has recently gained a lot of popularity due to the growing interest in multimedia applications. Signaling MIMO methods at wireless networks can provide two different form of improvement such as diversity methods and spatial multiplexing. Multiple paths between transmit and receive antennas provide greater robustness of the communication system than a monolithic method [15].

One of the biggest problems is the higher PAPR in OFDM. Consequently, it is capable of detecting nonlinear gadgets. With MIMO-OFDM, the PAPR information arrangement is made less auto correlative [18]. The MIMO-OFDM standard provides the most robust and flexible local area network and broadband access standards. When the transmitter does not have channel state information, MIMO-OFDM can still achieve particularly high capabilities [18].

An L-tap frequency-selective fading channel with $\mathrm{K}$ subcarriers, $\mathrm{N}$ transmits $\left(\mathrm{T}_{\mathrm{X}}\right)$ and $\mathrm{M}$ receives $\left(\mathrm{R}_{\mathrm{X}}\right)$ antennas, would signal over a system with $\mathrm{K}$ subcarriers. N OFDM transmitters are used in a MIMO-OFDM transmitter [16], in a serial-to-parallel converter (S/P), each input stream is grouped into $\mathrm{N}$ sequences including $\mathrm{KD}$ data symbols. During parallel operations, training symbols are inserted, inverse discrete Fourier transformation (IDFT) is performed, then it is up-converted to radio frequency (RF) centered at the fc point and the signal is transmitted. 


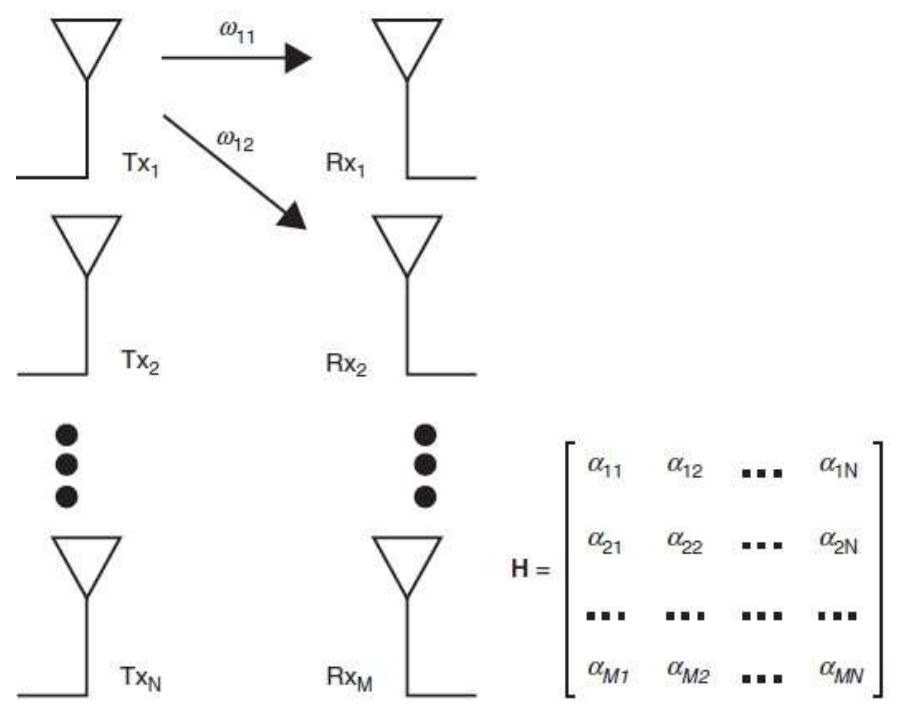

Figure 2. Array of transmitters and receivers and the resulting transfer matrix $\mathbf{H}$.

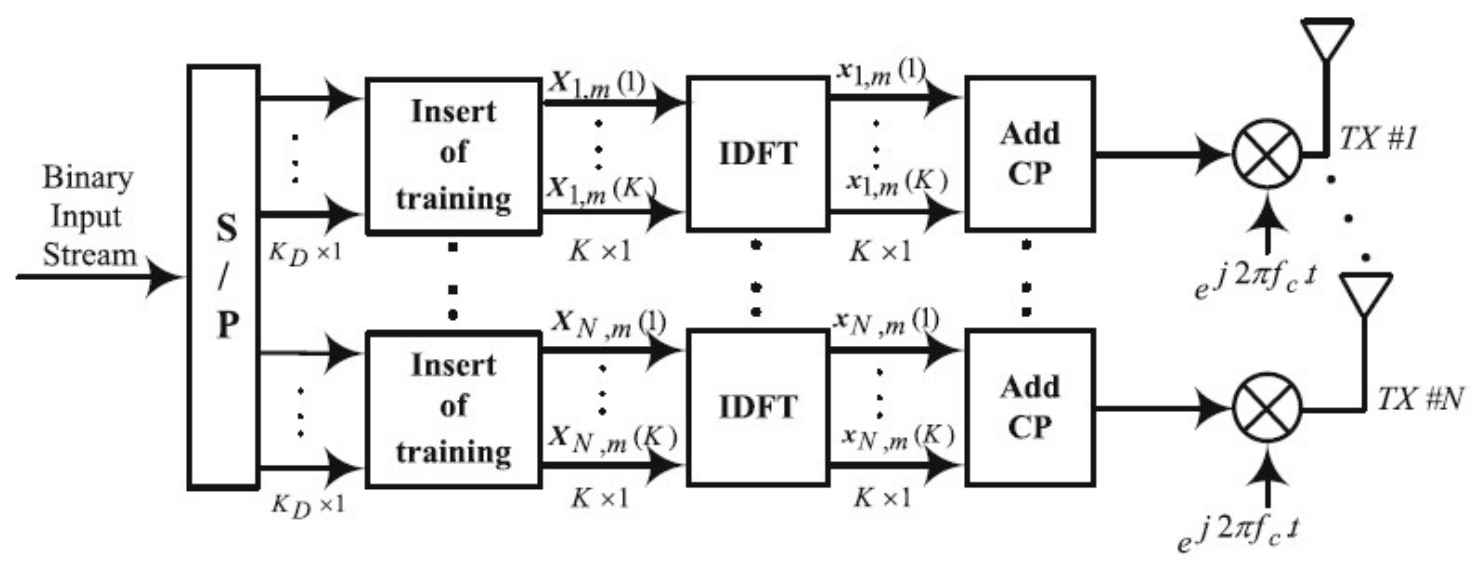

Figure 3. MIMO- OFDM system

\subsection{MIMO System Channel Capacity}

Signal fading is a major cause of multipath propagation. Diversification techniques have been developed to remedy this issue. Among the several forms of diversity, antenna diversity is widespread. Using multipath spread spectrum, multiple antennas can establish simultaneous parallel channels operating simultaneously on the same frequency band with the same power level, a result of multipath propagation theory. Various factors determine the antenna correlation: the scattering environment, the distance between the receiver and transmitter, the antenna configuration, and the Doppler spread. Multipath propagation has recently been proven to contribute to capacity in recent research. With a low probability of error at the receiver, channel capacity is the maximum rate of transmission and reception possible. Channel capacity is commonly measured in $\mathrm{bps} / \mathrm{Hz}$, which is a unit bandwidth of the channel [17]. 


\subsection{MIMO SYSTEM: MUTUAL COUPLING}

Antenna elements that are mutually coupled exhibit electromagnetic interactions. Fields generated by one antenna can alter voltage distributions at nearby antenna elements, especially if they are very close together. This is similar to how signal crosstalk in circuits disturbs the radiation pattern and input impedance of each element, because each element is affected by the other.

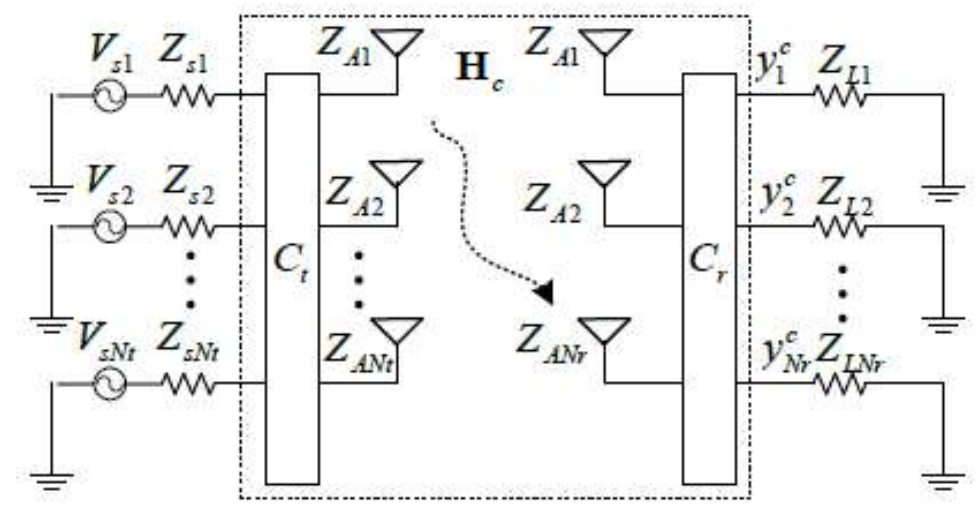

Figure 4. MIMO System with mutual coupling

\section{SIMULATION RESULTS}

Alamouti's scheme utilizes multiple antennas at a transmitter to deliver similar diversity gains. Its computation complexity is almost as great as that of the receive diversity system. According to figure 5, using two transmit antennas and one receive antenna provides the same diversity order as one transmit and two receive antennas arranged in maximal ratio combined (MRC). A simulation is performed for a system with and without channel estimation based on two receives antennas $(2 \times 2)$, shown in figure 6 . A simulation comparing the Alamouti code with or without antenna coupling for each SNR value was conducted. MIMO channel simulations are carried out with one Alamouti code each iteration, shown in figure 8 and figure 9.

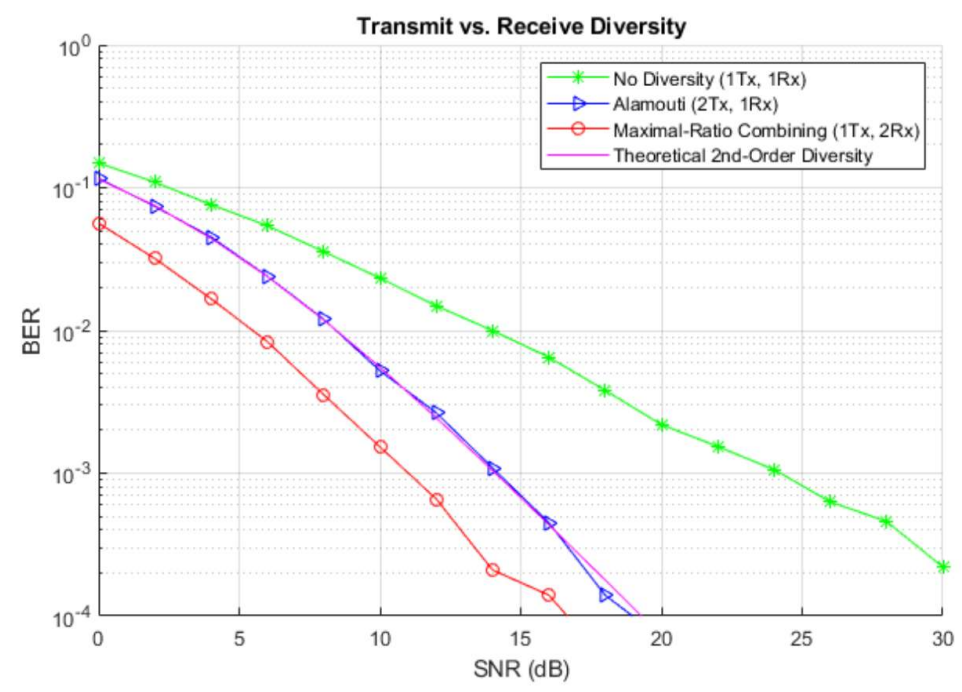

Figure 5. Transmit \& Receive Diversity 


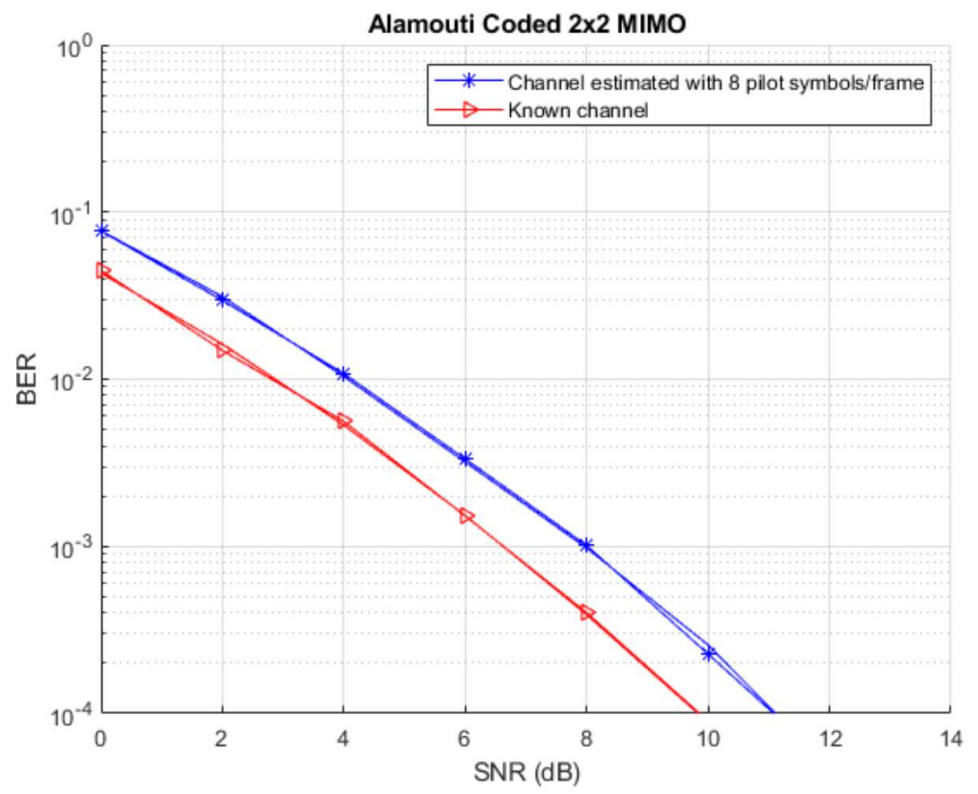

Figure 6. Alamouti coded 2x2 MIMO

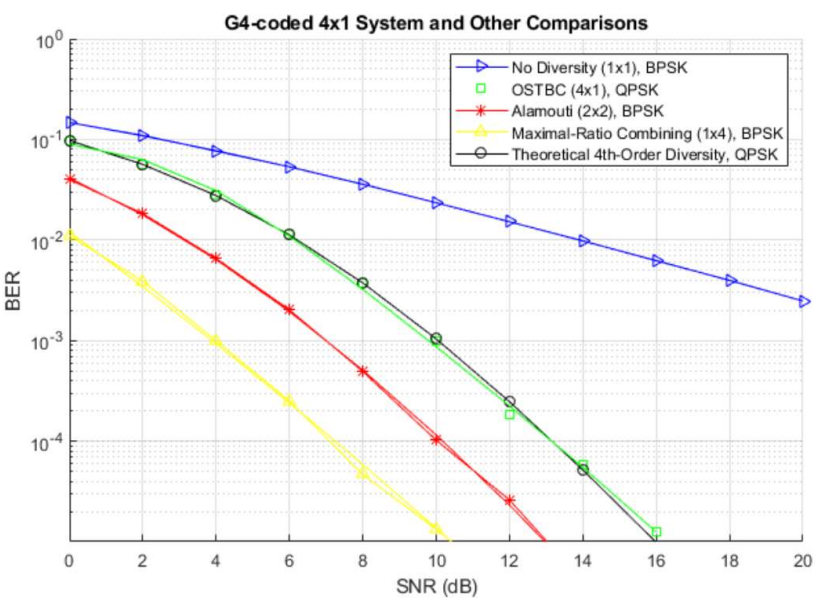

Figure 7. SNR vs BER for different MIMO systems

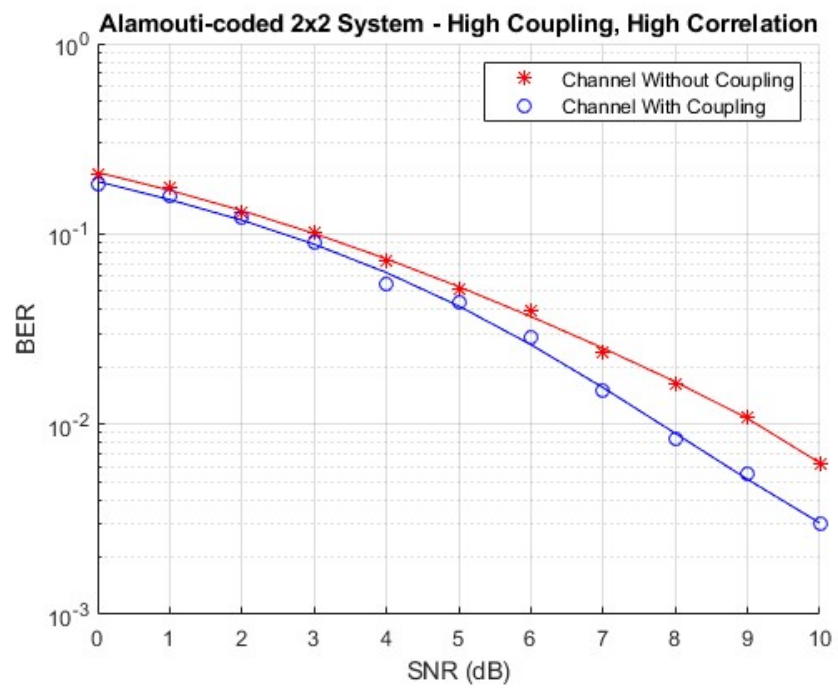

Figure 8. Alamouti Coded 2x2 MIMO with High Coupling and Correlation 


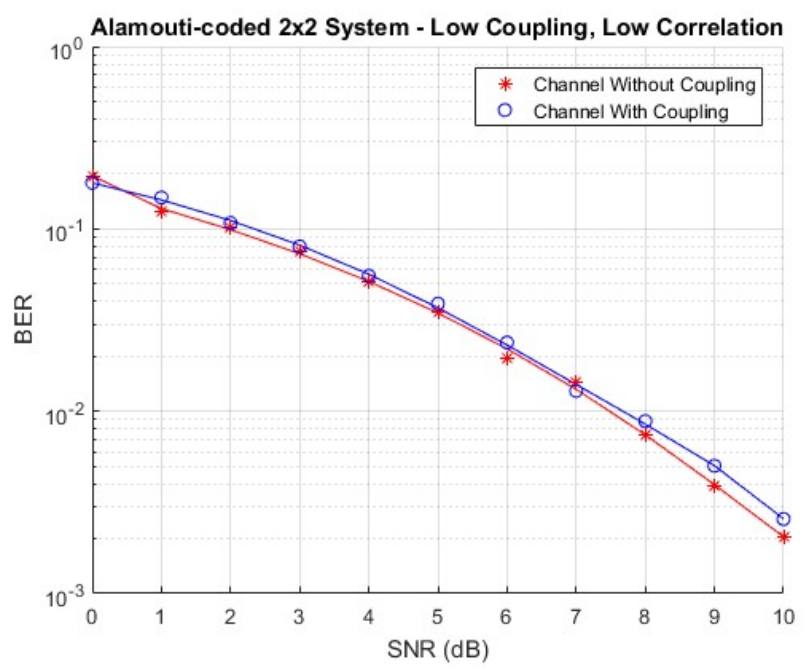

Figure 9. Alamouti Coded 2x2 MIMO with Low Coupling and Correlation

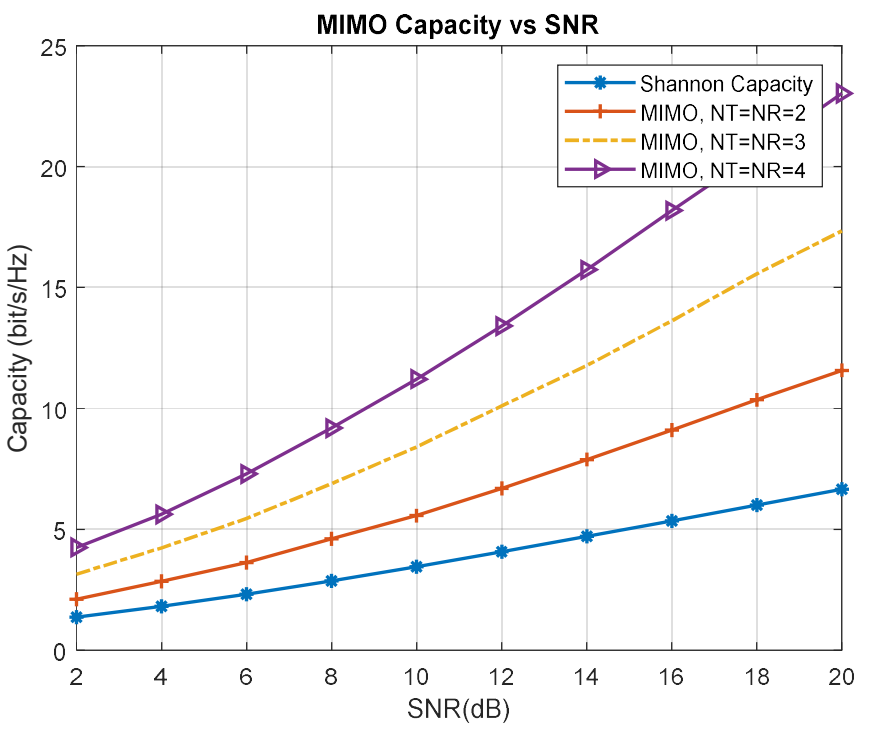

Figure 10. SNR vs Channel Capacity of MIMO

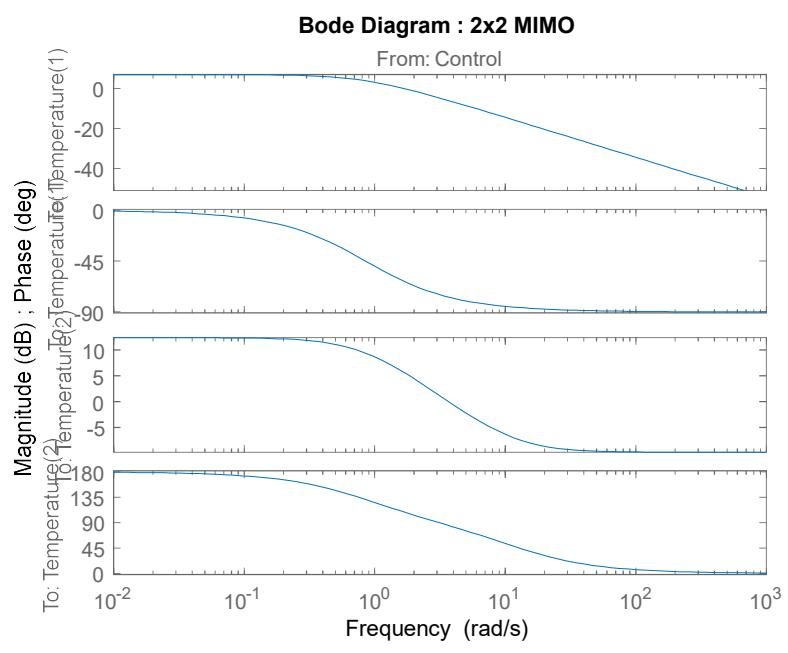

Figure 11. Frequency Response 


\section{CONCLUSION}

With the rapid increase in wireless data usage, wireless systems are challenged to allow for more bandwidth without requiring increased transmitter power. We have discussed a MIMO technology in this paper. The transmitter as well as the receiver uses more than one antenna in this technology, resulting in a noticeable increase in the wireless system's capacity. The effects of mutual coupling on MIMO antenna characteristics are demonstrated in this paper. In the numerical simulation, we find that signal power losses are large at small distances between elements, and the correlation coefficient becomes progressively smaller as the element distance increases. According to our results, the BER may be higher or lower depending on the correlation conditions.

\section{REFERENCES}

[1] S.Taruna and Ikpreet Kaur, "Performance Analysis of MIMO for Various Antenna Configurations", IEEE, 2013.

[2] RobertCalderbank, Anthony Constantinioles, Andera Goldsmith, Arogogyaswami Paulraj, H. Vincent Poor "Wireless Communication System”, Cambridge University Press 2007

[3] Muhammad Razin Ibn Azad "Multiple Input Multiple Output”, Thesis ,29thMay, 2012.

[4] G. J. Foschini, "Layered space-time architecture for wireless communication in a fading environment when using multi-element antennas," Bell Labs Tech. J., vol. 1, no. 2, pp. 41_59, Feb. 1996.

[5] F. Rusek et al., "Scaling up MIMO: Opportunities and challenges with very large arrays," IEEE Signal Process. Mag., vol. 30, no. 1, pp. 40_60, Jan. 2013.

[6] J. Zhang, X. Xue, E. Björnson, B. Ai, and S. Jin, "Spectral efficiency of multipair massive mimo two-way relaying with hardware impairments, "IEEE Wireless Commun. Lett., vol. 7 , no. 1, pp. 14_17, Feb. 2017.

[7] B. Ai et al., "On indoor millimeter wave massive MIMO channels: Measurement and simulation," IEEE J. Sel. Areas Commun., vol. 35, no. 7, pp. 1678_1690, Jul. 2017.

[8] Q. Yuan, Q. Chen, and K. Sawaya, "Performance of adaptive array antenna with arbitrary geometry in the presence of mutual coupling," IEEE Trans. Antennas Propag., vol. 54, no. 7, pp. 1991_1996, Jul. 2006.

[9] B. Wang, Y. Chang, and Y. Sun, "Performance of the large-scale adaptive array antennas in the presence of mutual coupling," IEEE Trans. Antennas Propag., vol. 64, no. 6, pp. 2236_2245, Jun. 2016.

[10] Y. Wu, J. W. M. Bergmans, and S. Attallah, "Effects of antenna correlation and mutual coupling on the carrier frequency offset estimation in MIMO systems," in Proc. Int. Conf. Wireless Commun. Netw. Mobile Comput. (WiCOM), Chengdu, China, Sep. 2010, pp. 144.

[11] S. Lu, H. T. Hui, M. E. Bialkowski, X. Liu, H. S. Lui, and N. V. Shuley, "The effect of antenna mutual coupling on channel estimation of MIMO-OFDM systems," in Proc. IEEE Antennas Propag. Soc. Int. Symp., Honolulu, HI, USA, Jun. 2007, pp. 1_4. 
[12] H. S. Lui and H. T. Hui, "Mutual coupling compensation for direction of arrival estimations using the receiving-mutual-impedance method," Int. J. Antennas Propag., vol. 2010, Jan. 2010, Art. no. 373061.

[13] Godara, L. C., Handbook of Antennas in Wireless Communications, 888, CRC Press, 2002.

[14] P. R O JSEL, "RF MEMS-based wireless architectures and front-ends", Woodhead Publishing Limited, 2013, DOI : 10.1533/9780857098610.1.207

[15] Arun Agarwal and Saurabh N. Mehta, "Design and Performance Analysis of MIMOOFDM System Using Different Antenna configurations", International Conference on Electrical, Electronics, and Optimization Techniques (ICEEOT) - 2016,

[16] S. Askar, S. Memon, L. Das and M. S. Kalhoro, -Impact of Modeling Different Fading Channels on Wireless MAN Fixed IEEE802.16d OFDM System with Diversity Transmission Techniquel, World academy of Science, Enginreering and Technology, vol. 5, pp. 917-923, 2011.

[17] Nirmalendu Bikas Sinha, Makar Chand Snai, M. Mitra, "Per formance Enhancement of MIMO-OFDM Technology for High Data Rate Wireless Networks", International Journal of Computer Science and Application Issue 2010.

[18] Karthik Kumar Vaigandla, Mounika Siluveru and Sandhya Rani Bolla, "Analysis of PAPR and Beamforming For 5 G MIMO-OFDM", International journal of analytical and experimental modal analysis, Volume XII, Issue X, 2020, pp.483-490.

[19] A. A. Abouda and S. G. H"aggman, "Effect Of Mutual Coupling On Capacity Of MIMO Wireless Channels In High Snr Scenario”, Progress In Electromagnetics Research, PIER 65, 27-40, 2006.

[20] Xiaoming Chen, Shuai Zhang and Qinlong Li, "A Review of Mutual Coupling in MIMO Systems", VOLUME 6, IEEE., 2018.

[21] Karthik Kumar Vaigandla, Allanki Sanyasi Rao and Kallepelli Srikanth, "Study of Modulation Schemes over a Multipath Fading Channels", International Journal for Modern Trends in Science and Technology 2021, 7 pp. 34-39. 